\title{
Computational Complexity and Peak-to-Average Power Ratio Reduction of OFDM Signals by PTS with Sub - optimum Grouping Phase Weighting Method
}

\author{
Prashant Pandey \\ Student Member, IEEE \\ Department of Electronics and \\ Communication, \\ Motilal Nehru National Institute \\ of Technology, Allahabad
}

\author{
Rajeev Tripathi \\ Member, IEEE \\ Professor, \\ Motilal Nehru National Institute \\ of Technology, Allahabad
}

\begin{abstract}
Partial Transmit Sequence (PTS) is a promising technique to reduce Peak-to-average power ratio (PAPR) of orthogonal frequency division multiplexing (OFDM) signals. However optimum PTS (OPTS) needs exhaustive search over all combinations of allowed phase weighting factors. It results in high computational complexity. Grouping Phase Weighting (GPW) technique is a method which has reduced computational complexity while same PAPR reduction capability as compared to O-PTS. In this paper we propose a novel technique known as Sub-optimum Grouping Phase Weighting (S-GPW) Method which not only reduces the computational complexity but also have an advantage of increment in PAPR reduction performance as compared to Grouping Phase Weighting (GPW) method.
\end{abstract}

\section{General Terms}

Orthogonal Frequency Division Multiplexing (OFDM) System.

\section{Keywords}

Complementary cumulative distribution function (CCDF), Partial Transmit Sequence (PTS), Peak -to-Average Power Ratio.

\section{INTRODUCTION}

Orthogonal frequency division multiplexing (OFDM) is a technique used for high-speed data transmission in wireless communication systems [1]. A major problem associated with orthogonal frequency division multiplexing (OFDM) is its large peak-to-average power ratio (PAPR), which degrades the system performance by introducing nonlinearity in the devices such as power amplifiers (PAs). In order to mitigate nonlinear distortion, linear high power amplifiers and analog to digital converters with a large dynamic range are required, but such power amplifiers are inefficient [2].

To reduce the PAPR of the OFDM signal, many techniques have been proposed in so far. These schemes can be classified into signal distortion schemes and signal scrambling schemes. The signal distortion schemes reduce high peaks directly by distorting the signal prior to amplification. Both clipping and companding techniques are typical signal distortion methods to lower PAPR [3], [4].However, these signal distortion schemes may cause large in-band and out-of-band distortions, resulting in the degradation of the system performance [5].Signal scrambling techniques are different in how to scramble the codes for the PAPR reduction. Some known scrambling techniques including selective mapping (SLM)
[5], partial transmit sequence (PTS) [6], and selective mapping of partial tones (SMOPT) [7]. Among all existing schemes, partial transmit sequences (PTS) is an important technique because of its good PAPR reduction performance without any signal distortion.

In this paper, we propose a phase weighting method for PTS, named as Sub-optimum grouping phase weighting method (SGPW) [8]-[10]. It not only reduces the computational complexity as compared to O-PTS and PTS with GPW but also it shows improved PAPR performance as compared to O-PTS and PTS with GPW.

This paper is organised as follows. In Section 2, we present the OFDM system model, definition of the PAPR and the definition of the CCDF. In Section 3, we have described optimal PTS and grouping phase weighting method for PTS. Section 4 introduces proposed Sub-optimum grouping phase weighting method (S-GPW) method [11]-13] for PTS. In section 5 , the performance of proposed phase weighting method is discussed and the simulation and numerical results are shown. Finally, we end the paper with brief conclusions in Section 6.

\section{PAPR IN OFDM SYSTEM}

\subsection{OFDM System Model}

In OFDM system, a block of $N$ symbols $X=\left\{X_{K}, k=0,1\right.$, ....., N-1 $\}^{\mathrm{T}}$ is formed with each symbol modulating one of a set of subcarriers, $\left\{\mathrm{f}_{\mathrm{k}}, \mathrm{k}=0,1,2, \ldots \ldots \ldots, \mathrm{N}-1\right\}$ where $\mathrm{N}$ is the number of subcarriers. The $\mathrm{N}$ subcarriers are chosen to be orthogonal, i.e. $f_{k}=k \Delta f$ where $\Delta f=1 /(N T)$ and $T$ is the original symbol period. Now, the complex envelope of the transmitted OFDM signals can be written as,

$$
x(t)=\frac{1}{\sqrt{N}} \sum_{k=0}^{N-1} X_{k} e^{j 2 \pi f_{k} t}, 0 \leq t \leq N T
$$

where $\mathrm{j}=\sqrt{-1}$

\subsection{PAPR in OFDM System}

In general, the PAPR of OFDM signals $x(t)$ is defined as the ratio of the maximum instantaneous power and its average power

$$
\operatorname{PAPR}[x(t)]=\frac{0 \leq t \leq N T}{P_{a v}}
$$


Where $\mathrm{P}_{\mathrm{av}}$ is the average power of $x(t)$ and is given by:

$$
\mathrm{P}_{\mathrm{av}}=\frac{1}{\mathrm{NT}} \int_{0}^{\mathrm{NT}}|\mathrm{x}(\mathrm{t})|^{2} \mathrm{dt}
$$

\subsection{The CCDF of the PAPR}

The complementary cumulative distribution function (CCDF) of the PAPR is the most frequently used performance measure for PAPR reduction techniques. The CCDF of the PAPR denotes the probability that the PAPR of a data block exceeds a given threshold $\gamma$. When the number of the subcarriers $N$ is relatively small, the CCDF expression of the PAPR of OFDM signals can be expressed as

$$
\operatorname{Prob}\{\text { PAPR }>\gamma\}=1-\left(1-\mathrm{e}^{-\gamma}\right)
$$

\section{OPTIMAL PTS (O-PTS) AND GPW FOR PTS}

\subsection{The PTS Technique}

In PTS, as shown in fig. 1, an input data block of $\mathrm{N}$ symbols is partitioned into $\mathrm{M}$ disjoint subblocks, which are transformed into $\mathrm{M}$ time-domain partial transmit sequences. These partial sequences are independently rotated by phase factors. The objective of this approach is to optimally combine the subblocks to obtain the time domain OFDM signals with the lowest PAPR.

\subsection{Mathematical Model for the O-PTS Technique}

In O-PTS, the input data sequence of an OFDM system with $\mathrm{N}$ subcarriers is firstly partitioned into $\mathrm{M}$ disjoint subblocks $\mathrm{X}_{\mathrm{i}}, \mathrm{i}=1,2, \ldots, \mathrm{M}$, where all the subcarriers which are occupied by the other subblocks are set to zero. The frequency domain input sequence is given as

$$
\mathrm{X}=\sum_{\mathrm{i}=1}^{\mathrm{M}} \mathrm{X}_{\mathrm{i}}
$$

By applying a phase weighting factor $\mathrm{b}_{\mathrm{i}}=\exp \left(\mathrm{j} \Phi_{\mathrm{i}}\right), \Phi_{\mathrm{i}} \in[0$, $2 \pi)$ to the $i^{\text {th }}$ subblock $X_{i}=\left[X_{i, 1}, X_{i, 2}, \ldots \ldots \ldots, X_{i, N}\right]^{T}, i=1,2$, ......, $\mathrm{M}$, alternative frequency signal sequence is given as

$$
X^{\prime}=\sum_{i=1}^{M} b_{i}
$$

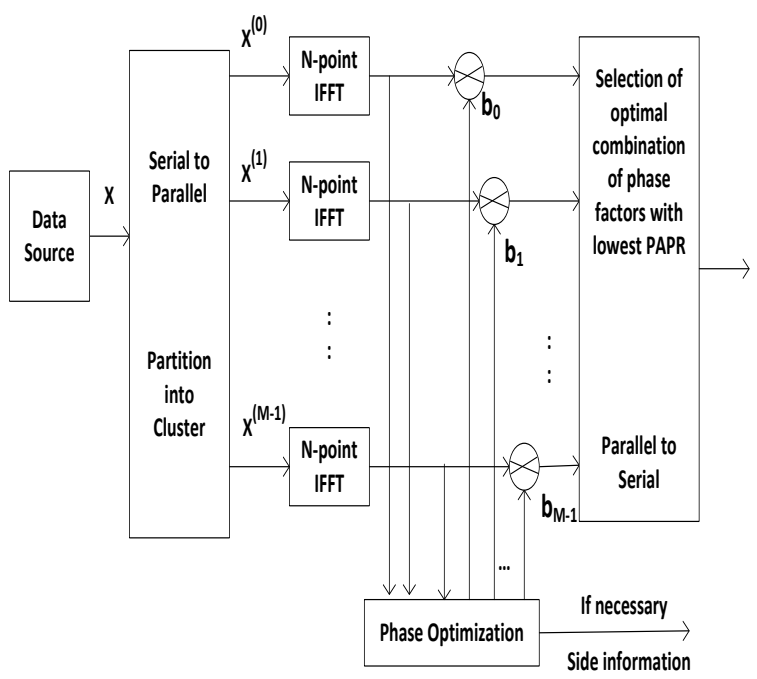

Figure 1: Block Diagram of the PTS Technique

After being transformed to time domain by IFFT, the time domain signal sequence becomes

$x^{\prime}=\operatorname{IFFT}\left\{\sum_{i=1}^{M} b_{i} X_{i}\right\}=\sum_{i=1}^{M} b_{i} \cdot \operatorname{IFFT}\left\{X_{i}\right\}=\sum_{i=1}^{M} b_{i} x_{i}$

In the above equation, $\mathbf{x}^{\prime}$ denotes the candidate sequence.

For generating different phase weighting sequences, a set of phase weighting factors is normally chosen. Assuming that there be $\mathrm{W}$ allowed phase weighting factors in this set. Without any loss of performance, we can set phase weighting factor for the first subblock to one and we see that there are (M-1) subblocks to be optimized. It is obvious that $\mathrm{W}^{\mathrm{M}-1}$ combinations must be checked to find the optimum candidate sequence with the minimum PAPR. For optimal PTS (OPTS), the optimum PAPR performance can be got after searching $\mathrm{W}^{\mathrm{M}-1}$ possible alternative combinations where $\mathrm{M}$ is the number of subblocks and $\mathrm{W}$ is the number of allowed phase weighting factors. In the process of phase weighting combination, large number of complex multiplications are needed and hence the computational complexity is very large.

3.3 Group Phase weighting (GPW) for PTS In order to reduce the computational complexity compared to O-PTS, GPW has been proposed in [8]. It offers same PAPR reduction performance with respect to O-PTS. In GPW, all the subblocks are split into several groups, and each group can obtain its own subcandiate sequence by using the same set of phase weighting factors; then, subcandidate sequences from different groups are combined to generate all the OFDM candidate sequences. Thereafter, that candidate sequence is selected for transmitting which has the minimum PAPR.

\section{SUB-OPTIMUM GROUPING PHASE WEIGHTING (S-GPW) METHOD}

The proposed approach has the mathematical model as follows: 


\subsection{Mathematical Model For the S-GPW Method}

The OFDM candidate sequence given in (7) can be written as

$$
\begin{gathered}
x^{\prime}=\sum_{i=1}^{M} b_{i} x_{i}=\sum_{i=1}^{r_{1}} b_{i} x_{i}+\sum_{i=r_{1}+1}^{r_{2}} b_{i} x_{i}+\cdots+\sum_{i=r_{R-1}+1}^{M} b_{i} x_{i} \\
1<r_{1}<r_{2} \ldots \ldots \ldots \ldots . r_{R-1}<V
\end{gathered}
$$

where the value of $r_{i}, i=1,2, \ldots \ldots ., R-1$ is the index of subblock. It can be observed in the above equation that all the subblocks can be divided into several groups, and for each group, phase weighting process can be implemented by itself. Here, we define $R$ to be number of groups, $G_{k}, k=1,2, \ldots, R$ to be the number of subcadidate sequence from the $\mathrm{k}^{\text {th }}$ group. The number of subblocks in each group can be expressed by $r_{1}, r_{2}-r_{1}, r_{3}-r_{2}, \ldots \ldots ., M-r_{R-1}$, where $r_{1}$ must be bigger that one (because phase weighting factor for the first subblock is constant). So Equation (8) can be written as

$$
\begin{aligned}
\mathrm{x}^{\prime}= & \sum_{\mathrm{i}=1}^{\mathrm{M}} \mathrm{b}_{\mathrm{i}} \mathrm{x}_{\mathrm{i}}= \\
& \sum_{\mathrm{i}=\mathrm{r}_{0}}^{\mathrm{r}_{1}} \mathrm{~b}_{\mathrm{i}} \mathrm{x}_{\mathrm{i}}+\sum_{\mathrm{i}=\mathrm{r}_{1}+1}^{\mathrm{r}_{2}} \mathrm{~b}_{\mathrm{i}} \mathrm{x}_{\mathrm{i}}+\ldots . .+\sum_{\mathrm{i}=\mathrm{r}_{\mathrm{R}-1}+1}^{\mathrm{r}_{\mathrm{R}}} \mathrm{b}_{\mathrm{i}} \mathrm{x}_{\mathrm{i}} \\
1= & \mathrm{r}_{0}<\mathrm{r}_{1}<\mathrm{r}_{2}<\ldots \ldots \ldots<\mathrm{r}_{\mathrm{R}-1}<\mathrm{r}_{\mathrm{R}}=\mathrm{M}
\end{aligned}
$$

By making use of the same set of phase weighting factors groups $\mathrm{G}_{\mathrm{i}}^{\text {'s }}, \mathrm{i}=1,2, \ldots \ldots, \mathrm{R}$ can implement their respective phase weighting processes and obtain their respective subcandidate sequences. Thereafter, subcandidate sequences from these different groups can be combined using complex additions to achieve all the OFDM candidate sequences, and the one with the minimum PAPR is selected for transmission.

We modify the above model such that within each group we implement sub-optimum PTS. The conjugate property of IDFT is used for all the odd subblocks (except the first one), increasing the number of the candidate signals equivalently. It can be obtained by performing the conjugate operation on all the odd subblocks (except the first one) before IDFT. By use of this linear property of IDFT, the transformed subblock is able to be obtained directly from the original one in the time domain instead of the use of IDFT. This avoids additional IDFTs and complex operations.

\section{The Property Used}

The OFDM signal is given by

$$
\boldsymbol{X}_{n}=\frac{1}{\sqrt{N}} \sum_{k=0}^{N-1} X_{k} e^{i 2 \pi k n / N}, 0 \leq n \leq N-1
$$

The transformed subblock is given by

$$
\begin{aligned}
& x_{n}=\frac{1}{N} \sum_{k=0}^{N-1}\left[X_{k}\right]^{*} \exp \left(j \frac{2 \pi}{N} n k\right) \\
& =\frac{1}{N} \sum_{k=0}^{N-1}\left[X_{k} \exp \left(-j \frac{2 \pi}{N} n k\right)\right]^{*} \\
& =\frac{1}{N} \sum_{k=0}^{N-1}\left[X_{k} \exp \left(j \frac{2 \pi}{N}(N k-n k)\right)\right]^{*} \\
& =\left[x_{N-n}\right]^{*}, k=0,1, \ldots ., N-1, n=0, \ldots \ldots, N-1
\end{aligned}
$$

In the proposed scheme alternate optimization and the linear property of IDFT are combined, where the use of alternate optimization results in reducing the computational complexity and the use of the linear property of inverse discrete Fourier transform (IDFT) is able to increase the number of candidate signals without any increase of the number of complex multiplications, which equivalently improves PAPR reduction performance. Hence the proposed scheme not only reduces the computational complexity but also show an improvement in PAPR reduction.

\section{PERFORMANCE ANALYSIS AND SIMULATION RESULTS}

\subsection{Performance Analysis}

The advantage in S-GPW is that it has lesser computational complexity than GPW and O-PTS and also the benefit of improved PAPR reduction capability than GPW and O-PTS.

\subsection{Simulation Results}

In this section, we do extensive simulations to verify the performance of the proposed schemes. For our simulation following parameters have been used.

Table 1 : Simulation Parameters

\begin{tabular}{|l|l|}
\hline $\begin{array}{l}\text { Number of OFDM blocks for } \\
\text { iteration }\end{array}$ & 3000 \\
\hline No. of Subcarriers (N) & $128,256,512$ \\
\hline No. of disjoint subblocks (M) & 4 \\
\hline Oversampling factor (L) & 4 \\
\hline Modulation type & QPSK \\
\hline No. of phase weighting factors & 2 \\
\hline The set of phase weighting factors & $\{1,-1\}$ \\
\hline
\end{tabular}


For PAPR reduction performance, the CCDF is used to evaluate and compare the performance of any PAPR reduction schemes. Figure 2, 3 and 4 show performance comparison between the original OFDM, S-GPW method and GPW method. As is Obvious from the figures S-GPW method has improved PAPR reduction capability than that of the GPW method which is the advantage as stated in the theoretical analysis. Also it is evident from the figures that PAPR reduction capability decreases as we increase the number of subcarriers.

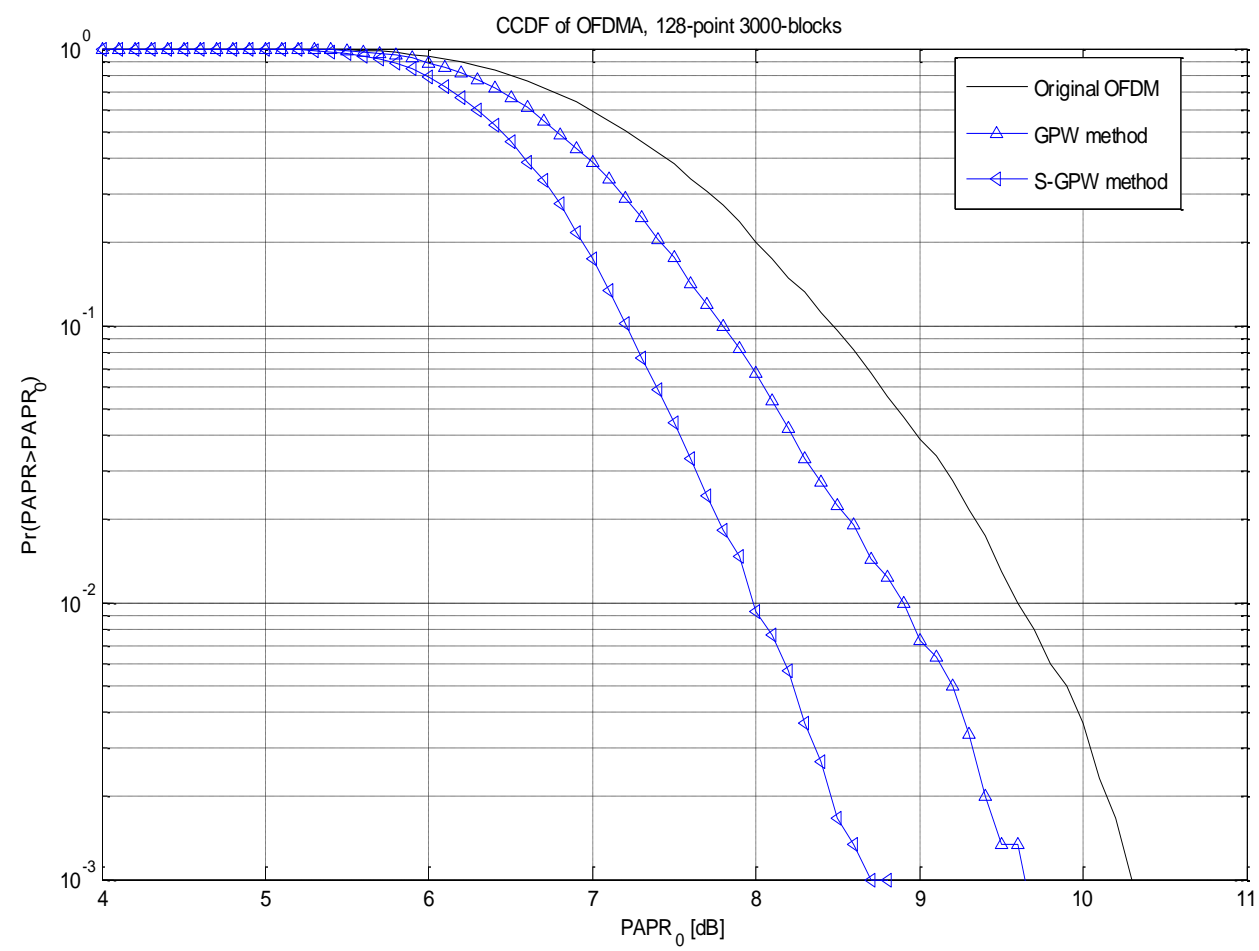

Figure 2: Comparison of CCDF of PAPR of PTS with S-GPW, GPW and Original OFDM Signal (N=128) 


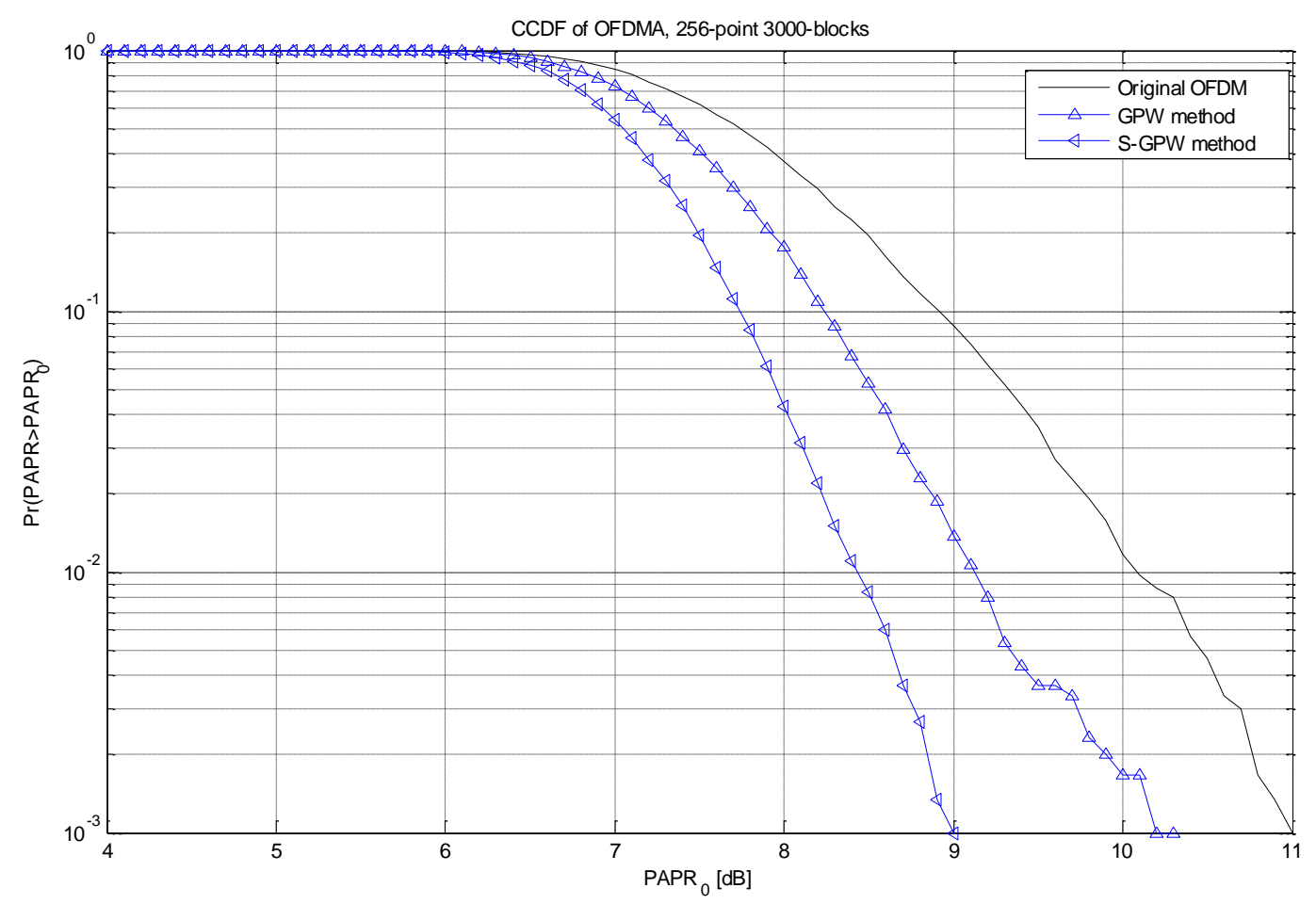

Figure 3: Comparison of CCDF of PAPR of PTS with S-GPW, GPW and Original OFDM Signal (N=256)

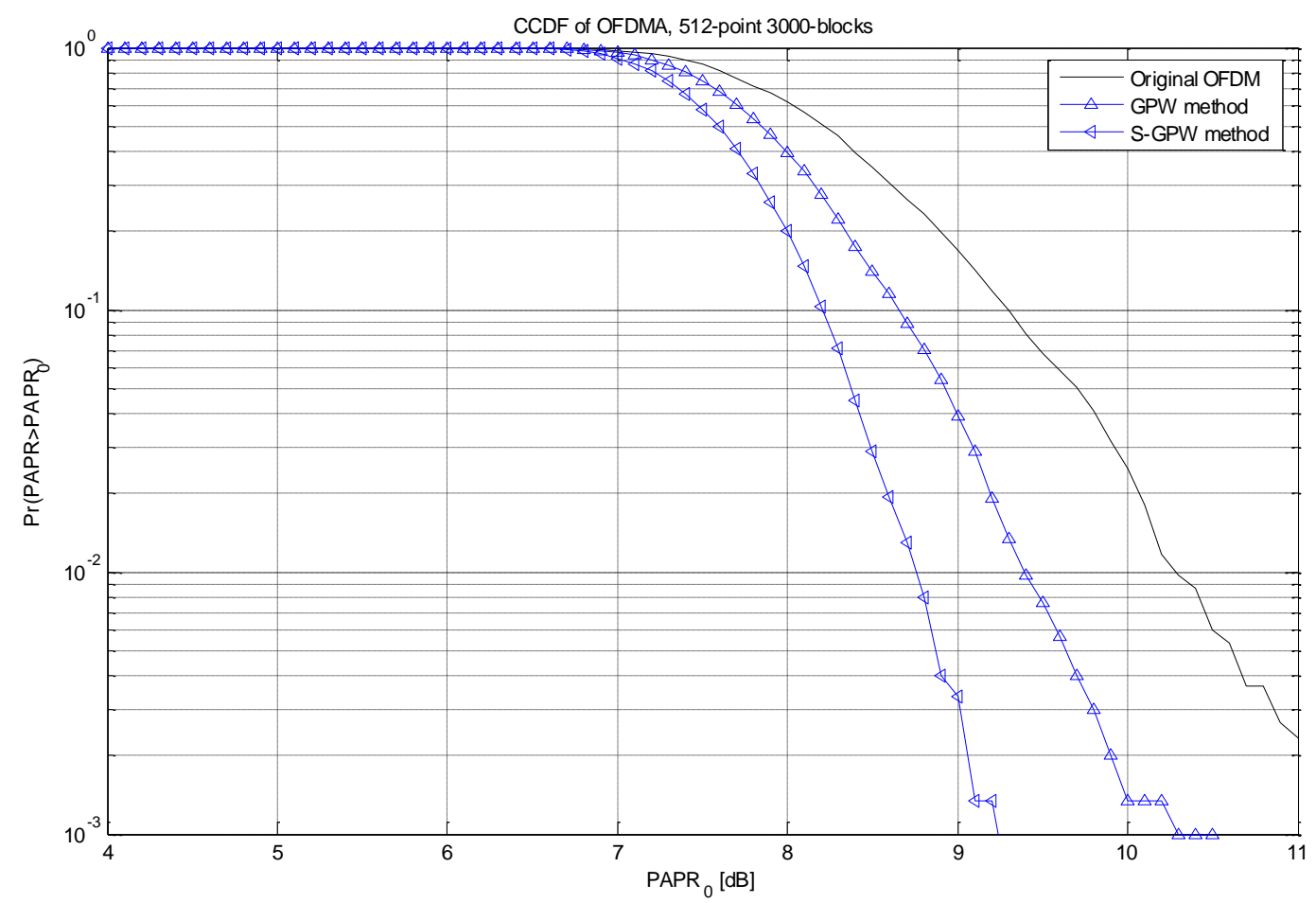

Figure 4: Comparison of CCDF of PAPR of PTS with AO-GPW, GPW and Original OFDM Signal (N=512) 


\section{CONCLUSIONS}

In this paper, a phase weighting method with low computational complexity and improved PAPR reduction performance for PTS is proposed. This method (S-GPW) has lesser computational complexity as compared to existing methods viz. GPW and O-PTS. Also it has the advantage that its PAPR reduction capability is greater as compared to GPW and O-PTS methods. Hence the computational complexity is reduced and also the PAPR reduction performance is improved.

\section{REFERENCES}

[1.] Zhu X., Jiang T., and Zhu G., "Novel Schemes Based on Greedy Algorithm for PAPR Reduction in OFDM Systems," IEEE Trans. on Consumer Electronics, Aug. 2008, 54, (3),

[2.] Kou Y., Lu W. S., and Antoniou A., "New Peak-toAverage Power-Ratio Reduction Algorithms for Multicarrier Communications," IEEE Trans. on and Systems, Sept 2004, 51, (9).

[3.] Li X. and Cimini, Jr. L. J., "Effect of Clipping and Filtering on the Performance of OFDM," IEEE Communication Letters, May 1998, 2, (5), pp. 131-133.

[4.] Jiang T. and Zhu. G. X., "Nonlinear Companding Transform for Reducing Peak-to-Average Power Ratio of OFDM Signals," IEEE Trans. on Broadcasting, Sept. 2004, 50, (3), pp. 342-346,

[5.] Bäuml, R. W. , Fisher R. F. H., and Huber J. B., "Reducing the Peak-to-Average Power Ratio of Multicarrier Modulation by Selected Mapping" Electronics Letters, Oct. 1996, 32, (22), pp. 2056-2057,

[6.] Müller S. H., Huber J. B., "OFDM with Reduced Peakto-Average Power Ratio by Optimum Combination of Partial Transmit Sequences," Electronics Letters, Feb. 1997, 33, (5), pp. 368-369.
[7.] S. Yoo, S. Yoon, S. Y. Kim, and I. Song, "A Novel PAPR Reduction Scheme for OFDM Systems: Selective Mapping of Partial Tones (SMOPT)", IEEE Trans. on Consumer Electronics, Feb. 2007, 52, (1), pp. 40-43.

[8.] Wang L. and Liu J., "PAPR Reduction of OFDM Signals by PTS With Grouping and Recursive Phase Weighting Methods," IEEE Trans. Broadcast., June 2011, 57, (2), pp. 1-8.

[9.] van Nee R. and Prasad R., OFDM for Wireless Multimedia Communications.

[10.]Wang L. and Cao Y., "Sub-optimum PTS for PAPR reduction of OFDM signals," Electron. Lett., Jul. 2008, 44, (15), pp. 921-922.

[11.]Pandey P. and Tripathi R., "Performance analysis of peak-to-average power ratio (PAPR) reduction techniques in an OFDM system," in Third IEEE International conference on computer and communication technology (ICCCT), Allahabad, pp. 245-249, Nov. 2012.

[12.]Pandey P. and Tripathi R., "Comparative Analysis of Peak-to-Average Power Ratio (PAPR) Reduction Techniques for OFDM System" in Fourth IEEE International conference on computer and communication technology (ICCCT), Allahabad, pp. 163-168, Sept. 2012

[13.]Pandey P. and Tripathi R., "Computational Complexity Reduction of OFDM Signals by PTS with Alternate Optimised Grouping Phase Weighting Method" International Journal of Computer Applications, Sept. 2013, 78, (1), pp. 1-7. 